\title{
Potential role of Nitric Oxide (NO) and Silver/ Silver Nanoparticles in the treatment of COVID-19 Infections
}

\author{
Tejvir Singh and Gordon McKaya* \\ Division of Sustainable Development, College of Science and Engineering, Hamad Bin Khalifa University, Qatar Foundation, \\ Qatar
}

\begin{abstract}
This short article provides an opinion on two potential opportunities that could be applied for the treatment of the virus COVID-19 and other similar viral pandemics that may attack humankind in the future. The two approaches are the use of nitric oxide and silver nanoparticles as therapeutic agents. This discussion focuses on the properties and characteristics of these two agents and why they present a potential treatment for COVID-19.
\end{abstract}

KEYWORDS: COVID-19 treatment; Nitric oxide; Silver-coated; Silver nanoparticles; Lipid layer destruction; Oxidative stress; Natural ways to increase nitric oxide levels inside the body

\section{INTRODUCTION}

The emergence of novel COVID-19 viral infection is causing ubiquitous fear in present times. Moreover, the viral spread of COVID-19 is not only generating a colossal economic loss for almost all countries but also the toll in precious human lives is at stake. Due to the unavailability of an approved vaccine or drug to treat the COVID-19 infection, in addition to the lack of sufficient testing protocols, the situation has been exacerbated further. This short discussion focuses on insights of the virus and potential solutions, which can be tried and tested to treat the patients affected by COVID-19.

\section{DISCUSSION}

The COVID-19 virus remains in a dormant phase unless it enters the living body, where after entering a living cell, it feeds on them by combining with angiotensin-converting enzyme 2(ACE 2) receptors. In this scenario, it can multiply itself exponentially.
Since no approved medicine is available in the market at this point of time, medical practitioners are forced to carry out symptoms management on the patients suffering from the virus. After studying the structure of the virus, it can be ratiocinated that the COVID 19 virus is fragile outside its body, which is protected by a thin layer of fat (lipids). Hence, one of the ways to kill the virus is by destroying its outer lipid layer, which can be used as a potential treatment approach. There are available disinfectants that can kill the virus outside the human body, such as hydrogen peroxide, chlorine, soap, alcohol-based sanitizers and disinfectants, available in the market. However, considering the toxicity of available disinfectants towards the human body, oxidation appears to be the best possible option inside the human body. However, if the oxygen content increases beyond $21 \%$, it will lead to free radical generation, and again the healthy cells will be destroyed. Consequently, selective dissolving of the virus fatty layer approach without affecting the healthy cells should be adopted.
Quick Response Code:

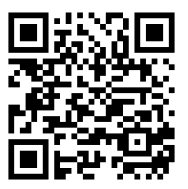

Address for correspondence: Gordon McKaya, Division of Sustainable Development, College of Science and Engineering, Hamad Bin Khalifa University, Qatar Foundation, Qatar

Received: May 12, 2020 Published: July 07, 2020

How to cite this article: Tejvir S, Gordon M. Potential role of Nitric Oxide (NO) and Silver/Silver Nanoparticles in the treatment of COVID-19 Infections. 2020 - 2(4) OAJBS.ID.000186. DOI: 10.38125/OAJBS.000186 
Nitric oxide (NO) is believed to offer multiple benefits. The presence of nitric oxide inside the human body and its effect on the human cardiovascular system was discovered by Robert F. Furchgott, Louis J. Ignarro, and Ferid Murad in the year 1998 and they shared the Noble Prize in 1998 for their discoveries concerning Nitric Oxide which serves as a signalling molecule in the cardiovascular system [1]. The use of nitric oxide in medical treatment received approval from the FDA (Food and Drug Administration) in the USA in the year 1999. Nitric oxide is naturally produced inside the human body through the consumption of dairy products, fresh green leafy vegetables, beets, Spirulina and citrus fruits like lemon, Indian gooseberry and mandarins. However, to increase the nitric oxide content inside the human body supplements such as L-arginine and L-citrulline can be used, both of which are amino acids and protein building blocks [2]. Nitric oxide has antimicrobial activity against bacteria, parasites, and fungi. The antiviral effects of Nitric Oxide are also known for mostly DNA viruses, including herpes simplex virus (HSV) and Epstein-Barr virus (EBV), murine poxvirus (ectromelia virus (ECTV)) and some RNA viruses such as coxsackievirus. EBV reactivation appears to be inhibited by Nitric Oxide via suppression of an immediate early-transactivator gene. Nitric oxide offers an oxidative effect on the cells, along with inhibiting the replication of viruses [3]. The most promising drugs for COVID-19 treatment are Hydroxychloroquine (C18H26ClN30) and Remdesivir (C27H35N608P). While comparing both these drugs, it can be concluded that Remdesivir offers inhibition of viral replication (chain termination) and reduction in virus RNA production, whereas, Hydroxychloroquine inhibits the stimulation of Tool-like receptors-9 (TLR-9) along with inhibiting the replication of several viruses. On the other hand, Nitric Oxide offers viral replication inhibition properties along with oxidation effects as required for the inactivation of viruses. Furthermore, nitric oxide can support better blood flow inside the human body; thus, the burden on ventilator requirements may ease significantly. The benefits of the nitric oxide treatment approach have been outlined in the recent trials carried out at Massachusetts General Hospital and the University of Alabama (Birmingham) [4-5].

The experimental trials of Nitric Oxide during 2004 SARS-CoV yielded promising results where Nitric Oxide enhanced severe hypoxia, reversed pulmonary hypertension, and reduced the mechanical ventilator support. The radiography images of the chest showed that the SARS spread was reduced and physiological effects stayed even after stopping the Nitric Oxide treatment [6]. Similar effects of Nitric Oxide application were also observed by Akerstrom et al. [7] where the research team concluded that Nitric Oxide inhibits the RNA synthesis as well as the viral protein of SARS virus which ultimately hinders the SARS-COV replication cycle. Considering all the treatment options used in COVID-19 treatment at present and the past trials with Nitric Oxide, it can be deduced that the oxidation effect created inside the human body has a significant role to play. This hypothesis is also supported by new research which is under trial and jointly carried out by the Indian Institute of Technology, Delhi (India) and the National Institute of Advanced Industrial Science and Technology, Japan. The team from these institutes is analyzing the role of compounds "Withanone (C28H38O6) and Caffeic Acid Phenethyl Ester (CAPE) (C17H1604)" for treating the COVID-19 infection as these compounds are believed to block the viral protein which is essential for replication of COVID-19 virus [8] Withanone is known for inducing oxidative stress and production of reactive oxygen species; hence the possibility to treat cancer has also studied with the use of Withanone for the same reason. On the other hand, CAPE is considered to have antiviral properties which preferentially destroy malignant cells [8]. Hence, the role of compounds known for inducing oxidative effect is crucial for the treatment of COVID-19.

Focusing on another possible protection in the treatment of COVID-19 viral infection is the application of silver nanoparticles. Several research papers have outlined the benefits that silver nanoparticles have successfully achieved mainly for their antimicrobial potential to fight against bacteria. However, they have also proven to be active against several types of viruses, including human immunodeficiency virus (HIV), Hepatitis B virus, Herpes simplex virus (HSV), Respiratory syncytial virus, and the monkeypox virus. The concept of using metals as a defense mechanism to neutralize the effect of microorganisms can be dated back to ancient times. The silver was a commonly utilized metal for culinary usage, along with preventing water contamination. Silver's mode of action includes the role of Ag+ ions, which severely restricts the growth of microorganisms by suppressing the electron transport mechanisms and respiratory enzymes and hence hinders the process of DNA functions. Therefore, silver was always considered useful as an antiviral, antibacterial, and antifungal metal. Moreover, silver, if consumed in approved concentrations found to be non-toxic for the human body. Also, it is believed that unlike antibiotics, the microorganisms are less likely to develop resistance against silver. Elechiguerra et al. [9] were the first to describe the antiviral activity of metal nanoparticles; in fact, they found that silver nanoparticles undergo size-dependent interactions with HIV1. The silver nanoparticles with 1-10 nm diameter coated in carbon and embedded in a foamy carbon matrix were able to bind to the virus. They had a greater inhibitory effect due to higher surface area availability of silver nanoparticles. Moreover, similar results have been observed with Herpes Simplex Virus-1 (HSV-1) where silver and gold nanoparticles blocked the attachment of the virus. Hence, the entrance of the virus inside the living cells was prohibited [10]. Xiang et al. [11] studied the effect of silver nanoparticles $(10 \mathrm{~nm}$ diameter) on the H1N1 influenza virus and reported that silver nanoparticles have anti-H1N1 influenza virus activities.

\section{CONCLUSION}

To conclude, it is expected that nitric oxide intake, along with the use of silver nanoparticles, will support the speedy recovery of patients from the viral infection of COVID-19. The suggested remedies will not have any negative ramifications on the health of the patient. They can be tested on some patients, followed by massscale implementation if the results are satisfactory. Furthermore, before using silver nanoparticles inside the human body, it will require trials and experiments along with regulatory approvals. However, until medical-grade silver nanoparticles are ready for permanent treatment of the virus, as an alternative to the silver nanoparticles dosage inside the human body, for the time being, the possibility of food intake through silver utensils can be tested for limiting the harmful effects of the virus on the human body as it is entirely safe, having been practised in culinary applications for many years.

\section{ACKNOWLEDGEMENT}

One of the authors is grateful to Hamad Bin Khalifa University for the provision of a research award. 


\section{REFERENCES}

1. Nicholls M (2019) Nitric oxide discovery noble prize winners. In Robert FF, Louis JI, Ferid M shared the noble prize in 1998 for their discoveries concerning nitric oxide as a signalling molecule in the cardiovascular system. European Heart Journal 40(22): 1747-1749.

2. (2019) Medical news today.

3. Akaike T, Maeda H (2000) Nitric oxide and virus infection. Immunology 101(3): 300-308.

4. Meredith S (2020) How the gas that gave the world Viagra could help treat coronavirus patients.

5. Pope A (2020) UAB among first in the US to offer a clinical trial for the treatment of patients with severe COVID-19 using nitric oxide.

6. Chen L, Liu P, Gao H, Sun B, Chao D, et al. (2004) Inhalation of nitric oxide in the treatment of severe acute respiratory syndrome: A rescue trial in Beijing. Clinical Infectious Diseases 39(10): 1531-1535.
7. Akerstrom S, Mousavi-Jazi M, Klingstrom J, Leijon M, Lundkvist A, et al. (2004) Nitric oxide inhibits the replication cycle of severe acute respiratory syndrome coronavirus. Journal of Virology 1966-1969.

8. Sari AN, Bhargava P, Dhanjal JK, Putri JF, Radhakrishnan N, et al. (2020) Combination of Withaferin-A and CAPE provides superior anticancer potency: Bioinformatics and experimental evidence to their molecular targets and mechanism of action. Cancers 12(5): 1160.

9. Elechiguerra JL, Burt JL, Morones JR, Camacho-Bragado A, Gao X (2005) Interaction of silver nanoparticles with HIV-1. Journal of Nanobiotechnology 29: 3-6.

10. Galdiero S, Falanga A, Vitiello M, Cantisani M, Marra V (2011) Silver nanoparticles as potential Antiviral Agents. MDPI-Molecules 16(10): 8894-8918.

11. Dong X, Qian C, Lin P, Cong LZ (2011) Inhibitory effects of silver nanoparticles on H1N1 influenza A virus in vitro. Journal of Virological Methods 178(1-2): 137-142. 